\title{
Nonholonomic Hybrid Zero Dynamics for the Stabilization of Periodic Orbits: Application to Underactuated Robotic Walking
}

\author{
Kaveh Akbari Hamed ${ }^{(\mathbb{1}}$ and Aaron D. Ames ${ }^{(}$
}

\begin{abstract}
This brief addresses zero dynamics associated with relative degree one and two nonholonomic outputs for exponential stabilization of given periodic orbits for hybrid models of bipedal locomotion. Zero dynamics manifolds are constructed to contain the orbit while being invariant under both the continuous- and discrete-time dynamics. The associated restriction dynamics are termed the hybrid zero dynamics (HZD). Prior results on the HZD have mainly relied on input-output linearization of holonomic outputs and are referred to as holonomic HZD (H-HZD). This brief presents reduced-order expressions for the HZD associated with nonholonomic output functions referred to as nonholonomic HZD (NH-HZD). This brief systematically synthesizes NH-HZD controllers to stabilize periodic orbits based on a reduced-order stability analysis. A comprehensive study of H-HZD and NH-HZD is presented. It is shown that NH-HZD can stabilize a broader range of walking gaits that are not stabilizable through traditional H-HZD. The power of the analytical results is finally illustrated on a hybrid model of a bipedal robot through numerical simulations.
\end{abstract}

Index Terms-Hybrid zero dynamics (HZD), nonholonomic outputs, periodic orbits, underactuated bipedal robots.

\section{INTRODUCTION}

$\mathbf{H}$ YBRID dynamical systems exhibit characteristics of both continuous- and discrete-time systems [1], [2] and have become a very common language to model legged locomotion [3]-[25]. Hybrid models of bipedal walking involve ordinary differential equations (ODEs) describing the Lagrangian continuous-time domains (i.e., phases) and discrete-time transitions describing the impact events [26]. Feedback controllers that deal directly with the hybrid nature of the bipedal robots have come out of hybrid reduction [27], [28], controlled symmetries [7], transverse linearization [9], [29], and hybrid zero dynamics (HZD) [4], [16], [30]. The notion of HZD was introduced in [30] to design feedback controllers that can explicitly accommodate underactuation in bipedal robots and move beyond quasi-static and flat-footed walking gaits. During the continuous-time domains, a set of parameterized output functions, referred

Manuscript received May 4, 2019; revised May 10, 2019 and August 30, 2019; accepted October 9, 2019. Manuscript received in final form October 10, 2019. The work of K. A. Hamed was supported by the National Science Foundation (NSF) under Grant 1854898, Grant 1906727, Grant 1923216, and Grant 1924617. The work of A. D. Ames was supported in part by NSF under Grant 1544332, Grant 1724457, Grant 1724464, Grant 1923239, and Grant 1924526, and in part by Disney Research LA. Recommended by Associate Editor M. Maggiore. (Corresponding author: Kaveh Akbari Hamed.)

K. A. Hamed is with the Department of Mechanical Engineering, Virginia Tech, Blacksburg, VA 24061 USA (e-mail: kavehakbarihamed@vt.edu).

A. D. Ames is with the Department of Mechanical and Civil Engineering, California Institute of Technology, Pasadena, CA 91125 USA (e-mail: ames@caltech.edu).

Color versions of one or more of the figures in this article are available online at http://ieeexplore.ieee.org.

Digital Object Identifier 10.1109/TCST.2019.2947874 to as virtual constraints [31]-[33], is defined and enforced by input-output (I-O) linearizing feedback controllers. The parameters of outputs are then updated by event-based laws during discrete-time transitions to render the associated zero dynamics manifolds hybrid invariant under the closed-loop dynamics [16]. HZD-based controllers have been validated numerically and experimentally for 2-D and 3-D bipedal robots [15], [34]-[39], powered prosthetic legs [40], [41], exoskeletons [42], monopedal [17], and quadruped robots [43]. The main advantage of the HZD approach is its systematic and geometric model reduction that significantly reduces the computational burden required for the motion planning and stabilization of periodic walking gaits for underactuated bipedal robots with high degrees of freedom. In particular, a periodic orbit of the HZD that lies in the zero dynamics manifolds is indeed an orbit of the full-order hybrid model. Therefore, one can apply the optimization-based motion planning algorithms to generate the orbit for the reduced-order HZD rather than the full-order model. In addition, if the feedback controllers render the zero dynamics manifolds attractive, the exponential stabilization of the periodic orbit for the HZD is equivalent to that of the orbit for the full-order hybrid system [6], [16], [44]. Hence, the stabilization problem of the periodic orbit can be investigated through a reduced-order Poincaré map rather than the full-order one.

\section{A. Motivation}

Although the vast body of work in HZD relies on zeroing uniform relative degree two holonomic outputs, nonholonomic outputs have also been utilized for the path planning of 3-D bipedal locomotion in [36] and [45]-[47]. Closed-form expressions for the HZD associated with holonomic virtual constraints, referred to as holonomic HZD (H-HZD), have been reported in [30] and [4, Ch. 6]. These results have been extended to 3-D walking gaits in [5], [37], and [44]. Although the generated gaits reported for nonholonomic outputs in [36] and [45]-[47] are stable, reduced-order stabilization problems have not been addressed for nonholonomic HZD (NH-HZD). In particular, the motion planning algorithms of [36] and [45] have been presented for the full-order hybrid systems rather than the reduced-order ones. Although [48] studied reduced-order NH-HZD for optimization-based gait planning of planar bipedal locomotion, the results are tailored to relative degree two nonholonomic outputs and not mixed relative degree one and two nonholonomic outputs. In addition, it does not address the stabilization and NH-HZD controller synthesis problems for given periodic orbits. In this brief, we aim to answer these questions: 1) how can we present closed-form solutions for relative degree one and two $\mathrm{NH}-\mathrm{HZD}$ to systematically reduce the computational burden 
for the exponential stabilization problem of given periodic orbits; 2) are there closed-form expressions for the restricted Poincaré maps; 3 ) if not, how can we effectively compute the restricted Poincaré maps; 4) how can we synthesize NH-HZD controllers that stabilize given walking gaits; and 5) are there nonholonomic virtual constraints to stabilize walking gaits that are not stabilizable through traditional holonomic outputs?

\section{B. Goals, Objectives, and Contributions}

The extension of the HZD approach to nonholonomic outputs is a challenge. First, unlike the H-HZD in [4] and [30], there is no closed-form expression for the solutions of the nonholonomic zero dynamics, which complicates the gait stability analysis. Second, nonholonomic zero dynamics manifolds are not, in general, invariant under the impact dynamics. This motivates the use of event-based update laws to ensure hybrid invariance, which, in turn, numerically complicates the gait stability analysis via the Poincaé return map. Third, the most important property of H-HZD, which is reduced-order gait stability analysis based on restricted Poincaré maps, is not easily extendable to the case of NH-HZD with event-based update laws. Although there are no closed-form expressions for the solutions of NH-HZD, this brief will show how NH-HZD controllers can stabilize a broader range of dynamic gaits. In particular, the stability of the gait for planar bipedal locomotion in the H-HZD approach depends solely on the gait trajectory and cannot be changed. More specifically, [4, Th. 6.2] states that the H-HZD approach fails to stabilize walking gaits for which the ratio of the value of the conjugate momentum around the stance leg end at the beginning of the step to that at the end of step is greater than or equal to one. This motivates the development of a systematic and computationally attractive approach to synthesize NH-HZD controllers that stabilize these dynamics gaits. The primary goal of this brief is to establish an analytical foundation to extend the HZD and reduced-order stabilization problem to hybrid models of planar bipedal robots with relative degree one and two nonholonomic outputs. The secondary goal is to present a systematic approach to design nonholonomic outputs that stabilize given underactuated walking gaits. These goals will be achieved through four objectives and contributions.

1) This brief presents closed-form expressions for NH-HZD with relative degree one and two outputs. These expressions are computationally important to develop the proposed controller synthesis approach based on restricted Poincaré maps. Two important classes of nonholonomic outputs are investigated. The first class consists of uniform relative degree two nonholonomic outputs. They are expressed in terms of the generalized positions as well as generalized conjugate momenta and are motivated by the work in [45]. The second class consists of mixed relative degree one and two nonholonomic outputs. Here, in addition to controlling the generalized positions and conjugate momenta, we regulate the forward walking velocity. The velocity-modulating quantities are motivated by the work on partial HZD (PHZD) in [36], [46], and [47].

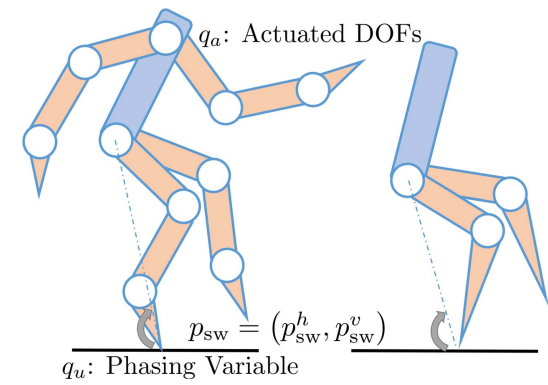

Fig. 1. Left: $N$ DOF model for planar and underactuated bipedal robots. Right: structure of the underactuated five DOF planar bipedal robot for the numerical simulation. A typical choice for the phasing variable is shown.

2) This brief extends the reduced-order stabilization approach that was originally developed for H-HZD [44] to that of periodic orbits lying in NH-HZD.

3) A comprehensive study of H-HZD and NH-HZD is presented to investigate their power in stabilizing given periodic orbits.

4) This brief finally shows how to synthesize stabilizing NH-HZD controllers. The power of the analytical results is numerically illustrated on a hybrid model of a bipedal robot.

\section{Hybrid Model of Bipedal Locomotion}

We shall suppose that the hybrid model of bipedal walking consists of one continuous-time domain, referred to as the single-support phase, plus a discrete-time transition, referred to as the instantaneous double-support phase. The underactuated and planar bipedal robot model is assumed to consist of a rigid tree structure with a torso link, two identical legs terminating at point feet and two possible identical arms (see Fig. 1). During the single-support phase, the mechanical system is supposed to have $N$ degrees of freedom with one degree of underactuation. In particular, the generalized coordinates can be partitioned as $q:=\operatorname{col}\left(q_{a}, q_{u}\right) \in \mathcal{Q}$, where $\mathcal{Q}$ represents the configuration space taken as an open and connected subset of $\mathbb{R}^{N}$. Moreover, $q_{a} \in \mathbb{R}^{N-1}$ and $q_{u} \in \mathbb{R}$ denote the actuated body (i.e., internal) and unactuated degrees of freedom, respectively. The evolution of the robot during the continuous-time domain is described by the Euler-Lagrange equations as follows:

$$
D(q) \ddot{q}+C(q, \dot{q}) \dot{q}+G(q)=B u
$$

in which $u \in \mathcal{U} \subseteq \mathbb{R}^{N-1}$ is the control input, $D(q) \in$ $\mathbb{R}^{N \times N}$ represents the positive definite mass-inertia matrix, and $B \in \mathbb{R}^{N \times(N-1)}$ denotes the input distribution matrix with the property $\operatorname{rank}(B)=N-1$. For future purposes, the centrifugal, Coriolis, and gravitational terms are lumped in the vector $H(q, \dot{q}):=C(q, \dot{q}) \dot{q}+G(q) \in \mathbb{R}^{N}$. The state vector for the control system (1) is taken as $x:=\operatorname{col}(q, \dot{q}) \in \mathcal{X}$, where $\mathcal{X}:=\mathrm{T} \mathcal{Q} \subset \mathbb{R}^{2 N}$ represents the state manifold. The state equation can be represented by $\dot{x}=f(x)+g(x) u$, where the vector filed $f(x)$ and columns of the $g(x)$ matrix are smooth (i.e., $\mathcal{C}^{\infty}$ ). In this brief, we shall consider walking on flat surfaces and assume that the instantaneous double-support phase occurs when the swing leg end contacts the ground. In particular, we define the guard of the hybrid system as $\mathcal{S}=\left\{x \in \mathcal{X} \mid p_{\mathrm{sw}}^{v}(q)=0, p_{\mathrm{sw}}^{h}(q)>0\right\}$, where $p_{\mathrm{sw}}:=$ 
$\operatorname{col}\left(p_{\mathrm{sw}}^{h}, p_{\mathrm{sw}}^{v}\right): \mathcal{Q} \rightarrow \mathbb{R}^{2}$ denotes the Cartesian coordinates of the swing leg end with respect to the ground. The evolution of the system during the double-support phase is then described by the following discrete-time model:

$$
x^{+}=\left[\begin{array}{c}
q^{+} \\
\dot{q}^{+}
\end{array}\right]=\left[\begin{array}{c}
\Delta_{q} q^{-} \\
\Delta_{\dot{q}}\left(q^{-}\right) \dot{q}^{-}
\end{array}\right]=: \Delta\left(x^{-}\right)
$$

where $x^{-}$and $x^{+}$denote the state of the system right before and after the discrete transition, respectively [4, Ch. 3], [26]. By integrating the continuous- and discrete-time dynamics, the hybrid model of locomotion can be expressed as

$$
\Sigma: \begin{cases}\dot{x}=f(x)+g(x) u, & x \in \mathcal{X} \\ x^{+}=\Delta\left(x^{-}\right), & x^{-} \in \mathcal{X} \cap \mathcal{S} .\end{cases}
$$

In this brief, we shall study periodic orbits to be exponentially stabilized. In particular, we make the following assumption.

Assumption 1 (Transversal and Periodic Orbit): There exist: 1) a fundamental period $T^{\star}>0$;2) a smooth and nominal control input $u^{\star}:\left[0, T^{\star}\right] \rightarrow \mathcal{U}$; and 3) a smooth and nominal state trajectory $\varphi^{\star}:\left[0, T^{\star}\right] \rightarrow \mathcal{X}$ such that $\dot{\varphi}^{\star}(t)=f\left(\varphi^{\star}(t)\right)+g\left(\varphi^{\star}(t)\right) u^{\star}(t)$ for all $0 \leq t \leq T^{\star}, \varphi^{\star}(t) \notin \mathcal{S}$ for every $0 \leq t<T^{\star}$ and $\varphi^{\star}\left(T^{\star}\right) \in \mathcal{S}$, and $\varphi^{\star}(0)=\Delta\left(\varphi^{\star}\left(T^{\star}\right)\right)$ (periodicity). Then, $\mathcal{O}:=\left\{x \in \mathcal{X} \mid x=\varphi^{\star}(t), 0 \leq t<T^{\star}\right\}$ is a period-one orbit of (3). We further suppose that $\mathcal{O}$ is transversal to $\mathcal{S}$. In particular, $\left\{x^{\star-}\right\}:=\overline{\mathcal{O}} \cap \mathcal{S}$ is a singleton, and $\dot{p}_{\text {sw }}^{v}\left(x^{\star-}\right) \neq 0$, where $\overline{\mathcal{O}}$ denotes the set closure of $\mathcal{O}$.

\section{NH-HZD FOR BIPEDAL WALKING}

The objective of this section is to address underactuated planar bipedal walking with nonholonomic outputs. We study two different output classes with relative degree one and two nonholonomic quantities. The restricted Poincaé map and controller synthesis problem for the stabilization of the orbit $\mathcal{O}$ will be addressed in Sections IV and V, respectivly.

Definition 1 (Nonholonomic Outputs): We will consider a family of parameterized nonholonomic outputs as follows:

$$
y=h(x, \alpha)=h(q, \dot{q}, \alpha)
$$

where $\operatorname{dim}(y)=\operatorname{dim}(u)=N-1$. Here, the output function is parameterized by the output parameters $\alpha \in \mathcal{A}$, where $\mathcal{A} \subseteq \mathbb{R}^{p}$ represents the set of admissible output parameters for some positive integer $p$. We remark that the reason for parameterization is to ensure that the corresponding zero dynamics manifolds are invariant under the discrete-time dynamics. This will be clarified more in Assumption 3.

Assumption 2 (Relative Degree): The family of output functions $h(x, \alpha)$ satisfies two properties: 1) there exists a nominal parameter, denoted by $\alpha^{\star} \in \mathcal{A}$, such that the nominal output function $h\left(x, \alpha^{\star}\right)$ vanishes on the given orbit $\mathcal{O}$, i.e., $h\left(x, \alpha^{\star}\right)=0$ for all $x \in \overline{\mathcal{O}}$ and 2) $h(x, \alpha)$ has relative degree vector $\left(r_{1}, \ldots, r_{N-1}\right)$ with respect to $u$ on an open neighborhood of $\mathcal{O}$, denoted by $\mathcal{N}(\mathcal{O}) \subset \mathcal{X}$. In particular, $\mathrm{L}_{g_{j}} \mathrm{~L}_{f}^{k} h_{i}(x, \alpha)=0$ for all $x \in \mathcal{N}(\mathcal{O}), \alpha \in \mathcal{A}$, $1 \leq i, j \leq N-1$, and $0 \leq k \leq r_{i}-2$ and the decoupling matrix $A(x, \alpha):=\left[\mathrm{L}_{g_{j}} \mathrm{~L}_{f}^{r_{i}-1} h_{i}(x, \alpha)\right]_{1 \leq i, j \leq N-1}$ is regular on $\mathcal{N}(\mathcal{O}) \times \mathcal{A} .^{1}$

\footnotetext{
${ }^{1}$ We remark that Assumption 2 is equivalent to having well-defined vector relative degree on the constraint manifold [31, Definition 3.1].
}

From Assumption 2 and [49, Ch. 5], one can design a $\mathcal{C}^{1}$ and parameterized $\mathrm{I}-\mathrm{O}$ linearizing feedback law on $\mathcal{N}(\mathcal{O}) \times \mathcal{A}$ as $u=\Gamma(x, \alpha)$ given by

$$
\Gamma(x, \alpha):=-A^{-1}(x, \alpha)(b(x, \alpha)+\ell(x, \alpha))
$$

to asymptotically zero the output function $h(x, \alpha)$ for the continuous-time dynamics, where $b(x, \alpha):=$ $\operatorname{col}\left\{\mathrm{L}_{f}^{r_{i}} h_{i}(x, \alpha)\right\}_{i=1}^{N-1}, \quad \ell(x, \alpha):=\operatorname{col}\left\{\ell_{i}(x, \alpha)\right\}_{i=1}^{N-1}, \quad$ and $\ell_{i}(x, \alpha):=\sum_{j=0}^{r_{i}-1}\left(K_{i, j} /\left(\epsilon^{r_{i}-j}\right)\right) \mathrm{L}_{f}^{j} h_{i}(x, \alpha)$ for $1 \leq i \leq$ $N-1$. In particular, employing the feedback law (5) yields the output dynamics $y_{i}^{\left(r_{i}\right)}+\sum_{j=0}^{r_{i}-1}\left(K_{i, j} /\left(\epsilon^{r_{i}-j}\right)\right) y_{i}^{(j)}=0$ for which the origin is assumed to be exponentially stable. Here, $K_{i, j}$ for $0 \leq j \leq r_{i}-1,1 \leq i \leq N-1$, and $\epsilon>0$ are positive scalars chosen such that the monic polynomials $\zeta^{r_{i}}+\sum_{j=0}^{r_{i}-1}\left(K_{i, j} /\left(\epsilon^{r_{i}-j}\right)\right) \zeta^{j}$ are Hurwitz. The adjustable parameter $\epsilon$ will be utilized for the singular perturbation analysis in Theorem 1. We remark that the control law (5) renders the parameterized zero dynamics manifolds $\mathcal{Z}_{\alpha}:=\left\{x \in \mathcal{X} \mid \mathrm{L}_{f}^{j} h_{i}(x, \alpha)=0,1 \leq i \leq\right.$ $\left.N-1,0 \leq j \leq r_{i}-1\right\}$ attractive and forward invariant under the flow of the closed-loop continuous-time dynamics $\dot{x}=f^{\mathrm{cl}}(x, \alpha)$, in which $f^{\mathrm{cl}}(x, \alpha):=f(x)+g(x) \Gamma(x, \alpha)$. From Assumption 2, $\operatorname{dim}\left(\mathcal{Z}_{\alpha}\right)=n_{z}:=n-r$ for all $\alpha \in \mathcal{A}$, where $r:=r_{1}+\cdots+r_{N-1}$. The evolution of the system restricted to the manifold $\mathcal{Z}_{\alpha}$ is further described by the parameterized zero dynamics $\dot{z}=f_{\text {zero }}(z, \alpha)$, where $z \in \mathbb{R}^{n_{z}}$ represents a set of local coordinates for $\mathcal{Z}_{\alpha}$.

\section{A. Relative Degree Two Nonholonomic Outputs}

In this section, we consider relative degree two nonholonomic outputs that are expressed in terms of the position variables and generalized conjugate momentum. In order to present the main idea, we make the following assumptions.

Hypothesis 1 (Phasing Variable): We assume that $q_{u}$ is a strictly increasing function of time along the desired periodic orbit $\mathcal{O}$, which is referred to as the phasing variable. We further suppose that $q_{u}$ is a cyclic variable for the continuous-time dynamics, that is, $\left(\partial D / \partial q_{u}\right)(q) \equiv 0$.

Next, let us consider the Lagrangian for the continuous-time dynamics as $\mathcal{L}(q, \dot{q}):=(1 / 2) \dot{q}^{\top} D(q) \dot{q}-V(q)$, where $V(q)$ represents the potential energy. We define the generalized conjugate momentum to $q_{u}$ as $\sigma:=\left(\partial \mathcal{L} / \partial \dot{q}_{u}\right)(q, \dot{q})=e_{N}^{\top} D(q) \dot{q}$, where $e_{N}=\operatorname{col}(0,0, \ldots, 1)$. From the Euler-Lagrange equations and the fact that $e_{N}^{\top} B=0$, one can rewrite the last row of (1) as $\dot{\sigma}=\left(\partial \mathcal{L} / \partial q_{u}\right)(q, \dot{q})$. Furthermore, Hypothesis 1 states that $\left(\partial \mathcal{L} / \partial q_{u}\right)=-\left(\partial V / \partial q_{u}\right)$. More specifically, $\left(\partial \mathcal{L} / \partial q_{u}\right)$ is solely a function of $q$, and therefore

$$
\dot{\sigma}=\frac{\partial \mathcal{L}}{\partial q_{u}}(q)=-\frac{\partial V}{\partial q_{u}}(q)=: \lambda(q) .
$$

We are now in a position to define the following parameterized and relative degree two nonholonomic output function:

$$
\begin{aligned}
4 y & :=h(x, \alpha):=h_{\mathrm{nom}}(x)-h_{\mathrm{corr}}\left(q_{u}, \alpha\right) \\
& :=\underbrace{q_{a}-q_{a}^{\star}\left(q_{u}\right)-h_{\sigma}(\sigma)+h_{\sigma}\left(\sigma^{\star}\left(q_{u}\right)\right)}_{=: h_{\mathrm{nom}}(x)}-h_{\mathrm{corr}}\left(q_{u}, \alpha\right)
\end{aligned}
$$


where $\operatorname{dim}(y)=\operatorname{dim}(u)=N-1$, and $q_{a}^{\star}\left(q_{u}\right)$ and $\sigma^{\star}\left(q_{u}\right)$ denote the desired evolutions of the actuated joint variables and conjugate momentum along the orbit $\mathcal{O}$ in terms of the phasing variable $q_{u}$, respectively. In addition, $h_{\sigma}: \mathbb{R} \rightarrow \mathbb{R}^{N-1}$ is a $\mathcal{C}^{\infty}$ function of $\sigma$ to be determined later. In particular, Section V will show that the proper selection of $h_{\sigma}$ would affect the stability behavior of the periodic orbit for the closed-loop hybrid system. We remark that for $h_{\sigma}(\sigma) \equiv 0$, the nonholonomic output (7) is reduced to a traditional holonomic output function. According to the construction procedure, the nominal output, $h_{\text {nom }}(x)$, vanishes on the desired orbit $\mathcal{O}$. Without loss of generality, we assume that the signs for the position and momentum terms in (7) are not same. The corrective term, $h_{\text {corr }}: \mathbb{R} \times \mathcal{A} \rightarrow \mathbb{R}^{N-1}$, is then defined as an additive term in (7) to zero the output function $y$ right after the impact event. One typical choice for the corrective term is a piceswise and sufficiently differentiable function of $q_{u}$ that is taken as a polynomial for the first half of the gait and zero for the second half [44, Example 1]. In this formulation, $\alpha \in \mathcal{A}$ denotes the coefficients of the polynomial. Therefore, one can choose the nominal parameters as zero, i.e., $\alpha^{\star}=0_{p}$, for which the corrective term vanishes. The following proposition addresses the 2-D zero dynamics.

Proposition 1 (Zero Dynamics for Relative Degree Two Outputs): Assume that Hypothesis 1 is satisfied. Consider the output function $y$ in (7) and rewrite it as

$$
y=h(x, \alpha)=h_{q}(q, \alpha)-h_{\sigma}(\sigma)
$$

in which $h_{q}(q, \alpha):=q_{a}-q_{a d}\left(q_{u}, \alpha\right)$ and $q_{a d}\left(q_{u}, \alpha\right):=$ $q_{a}^{\star}\left(q_{u}\right)-h_{\sigma}\left(\sigma^{\star}\left(q_{u}\right)\right)+h_{\text {corr }}\left(q_{u}, \alpha\right)$. Suppose further that the decoupling matrix $A(x, \alpha)=\frac{\partial h_{q}}{\partial q}(q, \alpha) D^{-1}(q) B$ is invertible on $\mathcal{N}(\mathcal{O}) \times \mathcal{A}$. Then, the following statements are correct.

1) Assumption 2 is satisfied. In particular, the output function $y=h(x, \alpha)$ has uniform relative degree 2 [i.e., $(2, \ldots, 2)]$ with respect to $u$ on $\mathcal{N}(\mathcal{O}) \times \mathcal{A}$.

2) $z(x):=\operatorname{col}\left(q_{u}, \sigma\right)$ is a valid tangent coordinates for the zero dynamics manifold $\mathcal{Z}_{\alpha}$. In addition, the position lift-up mapping on $\mathcal{Z}_{\alpha}$ is given by

$$
q=\pi_{q}\left(q_{u}, \sigma, \alpha\right):=\operatorname{col}\left(q_{a d}\left(q_{u}, \alpha\right)+h_{\sigma}(\sigma), q_{u}\right) .
$$

3) The zero dynamics can be expressed as

$$
\dot{z}=\left[\begin{array}{c}
\dot{q}_{u} \\
\dot{\sigma}
\end{array}\right]=f_{\text {zero }}(z, \alpha):=\left[\begin{array}{l}
\kappa_{1}\left(q_{u}, \sigma, \alpha\right) \\
\kappa_{2}\left(q_{u}, \sigma, \alpha\right)
\end{array}\right]
$$

where

$$
\begin{aligned}
4 \kappa_{1}\left(q_{u}, \sigma, \alpha\right) & :=\frac{\sigma-\varsigma\left(q_{u}, \sigma, \alpha\right)}{I\left(q_{u}, \sigma, \alpha\right)} \\
\kappa_{2}\left(q_{u}, \sigma, \alpha\right) & :=\left.\lambda(q)\right|_{q=\pi_{q}\left(q_{u}, \sigma, \alpha\right)}
\end{aligned}
$$

with $I\left(q_{u}, \sigma, \alpha\right):=D_{u u}(q)+D_{u a}(q)\left(\partial q_{a d} / \partial q_{u}\right)\left(q_{u}, \alpha\right)$ and $\varsigma\left(q_{u}, \sigma, \alpha\right):=D_{u a}(q)\left(\partial h_{\sigma} / \partial \sigma\right)(\sigma) \lambda(q)$ for $q=$ $\pi_{q}\left(q_{u}, \sigma, \alpha\right)$. In our notation

$$
D(q)=\left[\begin{array}{ll}
D_{a a}(q) & D_{a u}(q) \\
D_{u a}(q) & D_{u u}(q)
\end{array}\right]
$$

is a partitioning of $D$ corresponding to the coordinates vector $q=\operatorname{col}\left(q_{a}, q_{u}\right)$.
Proof: Differentiating the output function (8) results in $\dot{y}=\left(\partial h_{q} / \partial q\right) \dot{q}-\left(\partial h_{\sigma} / \partial \sigma\right) \lambda$ and $\ddot{y}=\left(\partial h_{q} / \partial q\right) \ddot{q}+$ $(\partial / \partial q)\left(\left(\partial h_{q} / \partial q\right) \dot{q}\right) \dot{q}-\left(\partial^{2} h_{\sigma} / \partial \sigma^{2}\right) \lambda^{2}-\left(\partial h_{\sigma} / \partial \sigma\right)(\partial \lambda / \partial q) \dot{q}$, which together with the system dynamics (1) yields $\ddot{y}=$ $A(x, \alpha) u+b(x, \alpha)$. Here, $A=\left(\partial h_{q} / \partial q\right) D^{-1} B$ and

$4 b=-\frac{\partial h_{q}}{\partial q} D^{-1} H+\frac{\partial}{\partial q}\left(\frac{\partial h_{q}}{\partial q} \dot{q}\right) \dot{q}-\frac{\partial^{2} h_{\sigma}}{\partial \sigma^{2}} \lambda^{2}-\frac{\partial h_{\sigma}}{\partial \sigma} \frac{\partial \lambda}{\partial q} \dot{q}$

which completes the proof of Part 1. Analogous to [30] and based on the proof of the Frobenius Theorem [49, p. 23], one can show that the tangent coordinates are given by $z=$ $\operatorname{col}\left(q_{u}, \sigma\right)$, and hence, Part 2 is correct. Finally, from (10), $\sigma=D_{u a} \dot{q}_{a}+D_{u u} \dot{q}_{u}$, which in combination with the position lift-up mapping and (6) completes the proof of Part 3.

\section{B. Relative Degree One and Two Nonholonomic Outputs}

In this section, we consider relative degree one and two nonholonomic outputs. In particular, we consider an output vector $y$ with the property $\operatorname{dim}(y)=\operatorname{dim}(u)=N-1$ and decompose it into relative degree one and two components as $y=\operatorname{col}\left(y_{d 1}, y_{d 2}\right)$. Here, $y_{d 1} \in \mathbb{R}$ and $y_{d 2} \in \mathbb{R}^{N-2}$ represent the relative degree one and two portions, respectively. The relative degree one portion (i.e., $y_{d 1}$ ) is assumed to be expressed in terms of the velocity, generalized conjugate momentum, and position variables, whereas the relative degree two portion (i.e., $y_{d 2}$ ) is supposed to be defined in terms of the position variables and generalized conjugate momentum analogous to the output function (7). Our motivation for considering a 1-D relative degree one portion [i.e., $\operatorname{dim}\left(y_{d 1}\right)=1$ ] is to regulate the stance hip forward velocity. This idea originates from the study of human-inspired control. In particular, by analyzing human locomotion data, [46] showed that the stance hip forward velocity appears to be a constant value. Since the relative degree two portion is $(N-2)$-dimensional (i.e., $\operatorname{dim}\left(y_{d 2}\right)=$ $N-2$ ), we consider a subset of actuated joint variables with $N-2$ components. More precisely, one can decompose the actuated variables as $q_{a}=\operatorname{col}\left(\tilde{q}_{a}, \breve{q}_{a}\right)$, where $\breve{q}_{a} \in \mathbb{R}^{N-2}$ represents the above-mentioned subset of $q_{a}$. Furthermore, $\tilde{q}_{a} \in \mathbb{R}$ denotes the actuated joint variable that is not being regulated as in (7). Instead, we regulate the forward walking speed of the robot. We are now in a position to define the parameterized output function $y$ as follows:

$$
\begin{aligned}
4 y:= & {\left[\begin{array}{l}
y_{d 1} \\
y_{d 2}
\end{array}\right]:=h(x, \alpha):=h_{\text {nom }}(x)-h_{\text {corr }}\left(q_{u}, \alpha\right) } \\
:= & \underbrace{\left[\begin{array}{r}
v-v^{\star}\left(q_{u}\right)+\mu\left(\tilde{q}_{a}-\tilde{q}_{a}^{\star}\left(q_{u}\right)-\tilde{h}_{\sigma}(\sigma)+\tilde{h}_{\sigma}\left(\sigma^{\star}\left(q_{u}\right)\right)\right) \\
\breve{q}_{a}-\breve{q}_{a}^{\star}\left(q_{u}\right)-\tilde{h}_{\sigma}(\sigma)+\tilde{h}_{\sigma}\left(\sigma^{\star}\left(q_{u}\right)\right)
\end{array}\right]}_{=: h_{\text {nom }}(x)} \\
& -\underbrace{\left[\begin{array}{l}
h_{\text {corr }, d 1}\left(q_{u}, \alpha\right) \\
h_{\text {corr }, d 2}\left(q_{u}, \alpha\right)
\end{array}\right]}_{=: h_{\text {corr }}\left(q_{u}, \alpha\right)}
\end{aligned}
$$

in which $v(q, \dot{q})$ represents the forward velocity of a point on the robot to be controlled (e.g., the stance hip), whose desired evolution on the gait is given by $v^{\star}\left(q_{u}\right)$. In $(11), \tilde{q}_{a}^{\star}\left(q_{u}\right) \in \mathbb{R}$, $\breve{q}_{a}^{\star}\left(q_{u}\right) \in \mathbb{R}^{N-2}$, and $\sigma^{\star}\left(q_{u}\right) \in \mathbb{R}$ denote the desired evolutions of $\tilde{q}_{a}, \breve{q}_{a}$, and $\sigma$ on the orbit, respectively. In addition, $h_{\sigma}(\sigma)=\operatorname{col}\left(\tilde{h}_{\sigma}, \breve{h}_{\sigma}\right)$ is a partitioning of the previously defined 
function $h_{\sigma}$ corresponding to the vector $q_{a}=\operatorname{col}\left(\tilde{q}_{a}, \breve{q}_{a}\right)$, i.e., $\tilde{h}_{\sigma} \in \mathbb{R}$ and $\breve{h}_{\sigma} \in \mathbb{R}^{N-2}$. The relative degree one portion then depends on a weighting factor $\mu \in \mathbb{R}$ as a tradeoff between controlling the forward velocity $v$ and controlling the actuated variable $\tilde{q}_{a}$. We remark that for $\mu=0$, one solely controls the forward velocity $v$. The corrective term can be decomposed as $h_{\text {corr }}\left(q_{u}, \alpha\right)=\operatorname{col}\left(h_{\text {corr }, d 1}, h_{\text {corr }, d 2}\right) \in \mathbb{R}^{N-1}$ compatible with the relative degree one and two components. The following proposition addresses the corresponding 3-D zero dynamics.

Proposition 2 (Zero Dynamics for Relative Degree One and Two Outputs): Assume that Hypothesis 1 is satisfied. Consider the output function $y$ in (11) and rewrite it as follows:

$$
y=\left[\begin{array}{c}
v(q, \dot{q})+\tilde{h}_{q}(q, \alpha)-\mu \tilde{h}_{\sigma}(\sigma) \\
\breve{h}_{q}(q, \alpha)-\breve{h}_{\sigma}(\sigma)
\end{array}\right]
$$

in which $\breve{h}_{q}(q, \alpha):=\breve{q}_{a}-\breve{q}_{a d}\left(q_{u}, \alpha\right)$ and

$$
\begin{aligned}
4 \tilde{h}_{q}(q, \alpha):= & -v^{\star}\left(q_{u}\right)+\mu\left(\tilde{q}_{a}-\tilde{q}_{a}^{\star}\left(q_{u}\right)+\tilde{h}_{\sigma}\left(\sigma^{\star}\left(q_{u}\right)\right)\right) \\
& -h_{\text {corr }, d 1}\left(q_{u}, \alpha\right) \\
\breve{q}_{a d}\left(q_{u}, \alpha\right):= & \breve{q}_{a}^{\star}\left(q_{u}\right)-\breve{h}_{\sigma}\left(\sigma^{\star}\left(q_{u}\right)\right)+h_{\mathrm{corr}, d 2}\left(q_{u}, \alpha\right) .
\end{aligned}
$$

The velocity $v$ can be expressed as $v(q, \dot{q})=J(q) \dot{q}$, where $J(q) \in \mathbb{R}^{1 \times N}$ is the corresponding Jacobian matrix. Suppose further that the parameterized decoupling matrix

$$
A(x, \alpha)=\left[\begin{array}{c}
J(q) \\
\frac{\partial \breve{h}_{q}}{\partial q}(q, \alpha)
\end{array}\right] D^{-1}(q) B \in \mathbb{R}^{(N-1) \times(N-1)}
$$

is invertible on $\mathcal{N}(\mathcal{O}) \times \mathcal{A}$. Then, the following statements are correct.

1) Assumption 2 is satisfied. In particular, the output function $y=h(x, \alpha)$ has relative degree $(1,2, \ldots, 2)$ with respect to $u$ on $\mathcal{N}(\mathcal{O}) \times \mathcal{A}$.

2) $z(x):=\operatorname{col}\left(q_{u}, \sigma, \tilde{q}_{a}\right)$ is a valid tangent coordinates for the manifold $\mathcal{Z}_{\alpha}$. Moreover, the position lift-up mapping on $\mathcal{Z}_{\alpha}$ is given by

$q=\pi_{q}\left(q_{u}, \sigma, \tilde{q}_{a}, \alpha\right):=\operatorname{col}\left(\tilde{q}_{a}, \breve{q}_{a d}\left(q_{u}, \alpha\right)+\breve{h}_{\sigma}(\sigma), q_{u}\right)$.

3) The zero dynamics can be expressed as

$$
\dot{z}=\left[\begin{array}{c}
\dot{\theta} \\
\dot{\sigma} \\
\dot{\tilde{q}}_{a}
\end{array}\right]=f_{\text {zero }}(z, \alpha):=\left[\begin{array}{l}
\kappa_{1}\left(q_{u}, \sigma, \tilde{q}_{a}, \alpha\right) \\
\kappa_{2}\left(q_{u}, \sigma, \tilde{q}_{a}, \alpha\right) \\
\kappa_{3}\left(q_{u}, \sigma, \tilde{q}_{a}, \alpha\right)
\end{array}\right]
$$

where $\kappa_{2}\left(q_{u}, \sigma, \tilde{q}_{a}, \alpha\right):=\lambda(q)$ for $q=\pi_{q}\left(q_{u}, \sigma, \tilde{q}_{a}, \alpha\right)$ and $\kappa_{1}$ and $\kappa_{3}$ are solutions of the following linear equation:

$$
\left[\begin{array}{ll}
I_{q_{u}} & I_{\tilde{q}_{a}} \\
J_{q_{u}} & J_{\tilde{q}_{a}}
\end{array}\right]\left[\begin{array}{l}
\kappa_{1} \\
\kappa_{3}
\end{array}\right]=\left[\begin{array}{c}
\sigma-\varsigma \\
-\tilde{h}_{q}+\mu \tilde{h}_{\sigma}-\omega
\end{array}\right] .
$$

In our notation, $I_{q_{u}}(z, \alpha):=D_{q_{u} q_{u}}(q)+$ $D_{q_{u} \breve{q}_{a}}(q)\left(\partial \breve{q}_{a d} / \partial q_{u}\right)\left(q_{u}, \alpha\right), \quad I_{\tilde{q}_{a}}(z, \alpha):=D_{q_{u} \tilde{q}_{a}}(q)$, and $\varsigma(z, \alpha):=D_{q_{u} \breve{q}_{a}}(q)\left(\partial \breve{h}_{\sigma} / \partial \sigma\right)(\sigma) \lambda(q)$, where

$$
D(q)=\left[\begin{array}{ccc}
D_{\tilde{q}_{a} \tilde{q}_{a}}(q) & D_{\tilde{q}_{a} \breve{q}_{a}}(q) & D_{\tilde{q}_{a} q_{u}}(q) \\
D_{\breve{q}_{a} \tilde{q}_{a}}(q) & D_{\breve{q}_{a} \breve{q}_{a}}(q) & D_{\breve{q}_{a} q_{u}}(q) \\
D_{q_{u} \tilde{q}_{a}}(q) & D_{q_{u} \breve{q}_{a}}(q) & D_{q_{u} q_{u}}(q)
\end{array}\right]
$$

is a partitioning of $D$ corresponding to the coordinates vector $q=\operatorname{col}\left(\tilde{q}_{a}, \breve{q}_{a}, q_{u}\right)$. Furthermore, $J_{q_{u}}(z, \alpha):=$

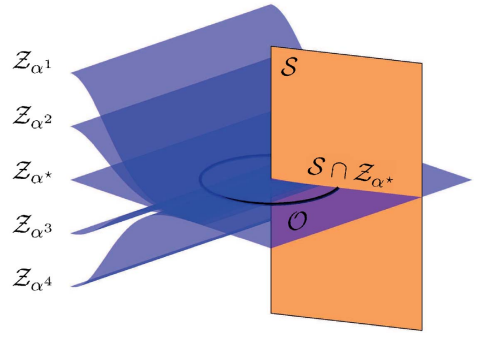

Fig. 2. Geometric illustration of the hybrid periodic orbit $\mathcal{O}$ and family of zero dynamics manifolds $\mathcal{Z}_{\alpha}$ for four different parameters $\alpha^{1}, \alpha^{2}, \alpha^{3}$, and $\alpha^{4} \in \mathcal{A}$. According to the construction procedure, $\mathcal{O} \subset \mathcal{Z}_{\alpha^{\star}}$ and $\mathcal{S} \cap \mathcal{Z}_{\alpha}=$ $\mathcal{S} \cap \mathcal{Z}_{\alpha^{\star}}$ for every $\alpha \in \mathcal{A}$.

$$
\begin{aligned}
& J_{q_{u}}(q)+J_{\breve{q}_{a}}(q)\left(\partial \breve{q}_{a d} / \partial q_{u}\right)\left(q_{u}, \alpha\right), J_{\tilde{q}_{a}}(z, \alpha):=J_{\tilde{q}_{a}}(q), \\
& \text { and } \omega(z, \alpha):=J_{\breve{q}_{a}}(q)\left(\partial \breve{h}_{\sigma} / \partial \sigma\right)(\sigma) \lambda(q) \text { for } \\
& q=\pi_{q}(z, \alpha) . \text { In our notation, } J(q) \text { = } \\
& \left.q J_{\tilde{q}_{a}}(q) J_{\breve{q}_{a}}(q) J_{q_{u}}(q)\right] \text { is also a partitioning } \\
& \text { of } J \text { corresponding to the coordinates vector } \\
& q=\operatorname{col}\left(\tilde{q}_{a}, \breve{q}_{a}, q_{u}\right) .
\end{aligned}
$$

Proof: Differentiating the output function $y$ results in $\dot{y}_{d 1}=J \ddot{q}+(\partial / \partial q)(J \dot{q}) \dot{q}+\left(\partial \tilde{h}_{q} / \partial q\right) \dot{q}-\mu\left(\partial \tilde{h}_{\sigma} / \partial \sigma\right) \lambda$, $\dot{y}_{d 2}=\left(\partial \breve{h}_{q} / \partial q\right) \dot{q}-\left(\partial \breve{h}_{\sigma} / \partial \sigma\right) \lambda$, and $\ddot{y}_{d 2}=\left(\partial \breve{h}_{q} / \partial q\right) \ddot{q}+$ $(\partial / \partial q)\left(\left(\partial \breve{h}_{q} / \partial q\right) \dot{q}\right) \dot{q}-\left(\partial^{2} \breve{h}_{\sigma} / \partial \sigma^{2}\right) \lambda^{2}-\left(\partial \breve{h}_{\sigma} / \partial \sigma\right)(\partial \lambda / \partial q) \dot{q}$, which in combination with the single-support phase dynamics (1) yields $\operatorname{col}\left(\dot{y}_{d 1}, \ddot{y}_{d 2}\right)=A(x, \alpha) u+b(x, \alpha)$. In particular, $A$ is given by (13) and

$b=\left[\begin{array}{c}\frac{\partial}{\partial q}(J \dot{q}) \dot{q}+\frac{\partial \tilde{h}_{q}}{\partial q} \dot{q}-\mu \frac{\partial \tilde{h}_{\sigma}}{\partial \sigma} \lambda \\ \frac{\partial}{\partial q}\left(\frac{\partial \breve{h}_{q}}{\partial q} \dot{q}\right) \dot{q}-\frac{\partial^{2} \breve{h}_{\sigma}}{\partial \sigma^{2}} \lambda^{2}-\frac{\partial \breve{h}_{\sigma}}{\partial \sigma} \frac{\partial \lambda}{\partial q} \dot{q}\end{array}\right]-\left[\begin{array}{c}J \\ \frac{\partial \breve{h}_{q}}{\partial q}\end{array}\right] D^{-1} H$ which completes the proof of Part 1 . Using the constructive proof the Frobenius Theorem [49, p. 23], we can show that the tangent coordinates are given by $z=\operatorname{col}\left(q_{u}, \sigma, \tilde{q}_{a}\right)$, and therefore, Part 2 is correct. From the definition of the angular momentum and forward velocity as well as the partitioning in (15), $\sigma=D_{q_{u}} \tilde{q}_{a} \dot{\tilde{q}}_{a}+D_{q_{u} \breve{q}_{a}} \dot{\tilde{q}}_{a}+D_{q_{u} q_{u}} \dot{q}_{u}$ and $v=J_{\tilde{q}_{a}} \dot{\tilde{q}}_{a}+$ $J_{\breve{q}_{a}} \dot{\grave{q}}_{a}+J_{q_{u}} \dot{q}_{u}$, which together with the position lift-up mapping and dynamics (6) completes the proof of Part 3.

\section{Reduced-Order Stabilization Problem}

The objective of this section is to present a reduced-order approach to address the exponential stabilization problem of the orbit $\mathcal{O}$ for (3) through restricted Poincaré maps. We extend the results of [44] to a broader range of HZD associated with nonholonomic outputs. In particular, the results of [44] were expressed for H-HZD with uniform relative degree outputs, whereas this section addresses NH-HZD. In our control strategy, the I-O linearizing controller generates attractive and invariant zero dynamics manifolds for the continuous-time domain. The event-based controller is then utilized to render these manifolds hybrid invariant for the closed-loop system. To clarify this idea, we make the following assumption.

Assumption 3 (Event-Based Update Law): We assume that there exists an event-based update law $\alpha^{+}=\gamma\left(x^{-}\right)$such that $\gamma\left(x^{\star-}\right)=\alpha^{\star}$; for every $\alpha \in \mathcal{A}$, the intersection $\mathcal{S} \cap \mathcal{Z}_{\alpha}$ 
is a nonempty and $\left(n_{z}-1\right)$-dimensional manifold that is independent of $\alpha$, i.e., $\mathcal{S} \cap \mathcal{Z}_{\alpha}=\mathcal{S} \cap \mathcal{Z}_{\alpha^{\star}}$ (see Fig. 2); and for every $x^{-} \in \mathcal{N}\left(x^{\star-}\right) \cap \mathcal{S} \cap \mathcal{Z}_{\alpha^{\star}}, x^{+}=\Delta\left(x^{-}\right) \in \mathcal{Z}_{\gamma\left(x^{-}\right)}$.

The following theorem extends the reduced-order stabilization problem of periodic orbits of H-HZD [16], [44] to that of periodic orbits of NH-HZD.

Theorem 1 (Reduced-Order Stabilization for NH-HZD): Under Assumptions 1-3, the following statements are correct.

1) Singular Perturbation and HZD: There is an $\bar{\epsilon}>0$ such that for all $0<\epsilon<\bar{\epsilon}$, the orbit $\mathcal{O} \times\left\{\alpha^{\star}\right\}$ is exponentially stable for the full-order model if and only if its projection onto $z$, i.e., $\mathcal{O}_{\text {zero }} \times\left\{\alpha^{\star}\right\}$, is exponentially stable for the following reduced-order NH-HZD:

$$
\Sigma_{\mathscr{N} \mathscr{H}-\mathscr{H} \mathscr{Z} \mathscr{D}}:\left\{\begin{array}{l}
{\left[\begin{array}{l}
\dot{z} \\
\dot{\alpha}
\end{array}\right]=\left[\begin{array}{c}
f_{\text {zero }}(z, \alpha) \\
0
\end{array}\right], \quad z^{-} \notin \mathcal{S}_{\text {zero }}} \\
{\left[\begin{array}{l}
z^{+} \\
\alpha^{+}
\end{array}\right]=\left[\begin{array}{c}
\Delta_{\text {zero }}\left(z^{-}\right) \\
\gamma_{\text {zero }}\left(z^{-}\right)
\end{array}\right], \quad z^{-} \in \mathcal{S}_{\text {zero }}}
\end{array}\right.
$$

where $\Delta_{\text {zero }}(z)$ and $\gamma_{\text {zero }}(z)$ represent the restriction of the impact map and event-based law to $\mathcal{S}_{\text {zero }} \cap \mathcal{Z}_{\alpha^{\star}}$, respectively. In addition, $\mathcal{S}_{\text {zero }}$ denotes the projection of the guard $\mathcal{S}$ onto the $z$-coordinates.

2) Reduced-Order Stability Criterion: The orbit $\mathcal{O}_{\text {zero }} \times$ $\left\{\alpha^{\star}\right\}$ is exponentially stable for the NH-HZD if and only if the eigenvalues of the Jacobian matrix $\mathrm{D}_{z} P_{\text {zero }}\left(z^{\star-}\right):=\left(\partial P_{\text {zero }} / \partial z\right)\left(z^{\star-}\right)$ strictly lie inside the unit circle, where $P_{\text {zero }}: \mathcal{S}_{\text {zero }} \cap \mathcal{Z}_{\alpha^{\star}} \rightarrow \mathcal{S}_{\text {zero }} \cap \mathcal{Z}_{\alpha^{\star}}$ represents the restriction of the Poincaré return map to $\mathcal{S}_{\text {zero }} \cap \mathcal{Z}_{\alpha^{\star}}$.

Remark 1: Theorem 1 follows from [44, Ths. 1 and 2] that is an extended version of [16, Corollary 11]. This result will be utilized in Section $\mathrm{V}$ for the proper selection of the function $h_{\sigma}(\sigma)$ that stabilizes the given gait. For this purpose, we will parameterize the function $h_{\sigma}(\sigma)$ with a finite-dimensional parameter vector $\xi$. Then, [44, Ths. 1 and 2] allows us to use the reduced-order Poincaré map for the HZD with adjustable and stabilizing parameters $\xi$, whereas [16, Corollary 11] does not consider this parameterization (see [44, Remark 3]).

Remark 2 (Advantage of NH-HZD): Unlike [30], there are no closed-form expressions for the state solutions of the NH-HZD. In particular, H-HZD consists of separable ODEs [4, Sec. 5.4.1] for which one can easily compute the flow map. However, those in (9) and (14) are not separable ODEs. Furthermore, we can show that, in general, the impact invariance condition cannot be achieved for nonholonomic zero dynamics manifolds unless event-based update laws are utilized. More specifically, [4, Th. 6.2] constructs holonomoic outputs for a given periodic orbit such that the corresponding zero dynamics manifolds are hybrid invariant. This result cannot be extended to the nonholonomic case, which, in turn, complicates the reduced-order stability analysis. However, thanks to Theorem 1, we can still have a reduced-order stabilization approach for NH-HZD. This will be clarified more in Section V. We will also show that zeroing nonholonomic outputs can stabilize periodic walking gaits that are not stabilizable through zeroing holonomic outputs. More specifically, [4, Th. 6.2] states that the H-HZD approach fails to stabilize walking gaits for which the ratio of the value of the momentum $\sigma$ at the beginning of the step to that at the end of step is greater than or equal to one, i.e., $\left(\sigma^{\star+} / \sigma^{\star-}\right) \geq 1$. Section $\mathrm{V}$ will numerically show how to synthesize NH-HZD controllers to stabilize these gaits.

Remark 3: In this brief, we only regulate one velocity-modulating output. The results of this brief can be extended to NH-HZD with multiple relative degree one outputs. However, by increasing the number of velocity-modulating outputs, the dimension of the zero dynamics manifolds becomes bigger. This increases the computational burden for the Jacobian linearization of the restricted Poincaré map.

\section{NUMERICAL SYNTHESIS OF NH-HZD CONTROLLERS FOR STABILIZATION OF Given PERIOdiC ORbITS}

The objective of this section is to apply the reduced-order approach of Theorem 1 to: 1) investigate the stability of given walking gaits for NH-HZD and 2) improve the stability behavior through the proper selection of the smooth function $h_{\sigma}(\sigma)$. We numerically show that the gaits that are not stabilizable through the traditional H-HZD can be stabilized with NH-HZD. We consider a robot structure with point feet as shown on the right-half of Fig 1. Each leg of the robot is assumed to include two actuated DOFs: a one-DOF hip joint plus a one-DOF knee joint. During the single-support phase, the robot has five DOFs, including four actuated DOFs of two legs and one unactuated DOF corresponding to the pitch motion of the torso link. The kinematic and dynamic parameter values for the links are taken according to those reported in [50] from a human cadaver study. Here, $q_{a}$ and $\tilde{q}_{a}$ are chosen as $q_{a}:=\operatorname{col}\left(q_{\mathrm{STK}}, q_{\mathrm{STH}}, q_{\mathrm{SWK}}, q_{\mathrm{SWH}}\right)$ and $\tilde{q}_{a}:=q_{\mathrm{STK}}$, where the subscripts "STK," "STH," "SWK," and "SWH" stand for the stance knee, stance hip, swing knee, and swing hip, respectively. The phasing variable $q_{u}$ in Hypothesis 1 is also chosen as the angle of the virtual leg with respect to the ground, in which the virtual leg is defined as the virtual line connecting the stance foot to the stance hip. Using the motion planning algorithm of [37], a desired periodic walking gait $\mathcal{O}$ is designed for walking at $0.7(\mathrm{~m} / \mathrm{s})$ with the cost of mechanical transport CMT $=0.17$ such that the instability criterion of Remark 2 is satisfied. This condition violates the necessary and sufficient conditions of $[4$, Th. 6.2] that are required for the exponential stabilization of the periodic orbit using the H-HZD approach. To confirm the instability of the gait, we set $h_{\sigma}(\sigma) \equiv 0$ in the uniform relative degree two output function (7) and then apply Theorem 1 to compute the derivative of the 1-D restricted Poincaré map as $\mathrm{D}_{z} P_{\text {zero }}\left(z^{\star-}, 0\right)=1.1006$. In particular, we make use of [44, Corollary 1] for the numerical computation of the Jacobian matrix. Fig. 3 illustrates the evolution of the pitch angular velocity versus time during 25 consecutive steps of walking for which the initial condition has been chosen off of the orbit. The state trajectory diverges from the orbit.

To stabilize the gait, we now consider a linear family ${ }^{2}$ for $h_{\sigma}(\sigma)$ that is parameterized by a set of parameters as

\footnotetext{
${ }^{2}$ Using the analytical approach of this brief, one can also consider other smooth functions for $h_{\sigma}$. However, to simplify the analysis, this section presents numerical results for a linear function.
} 

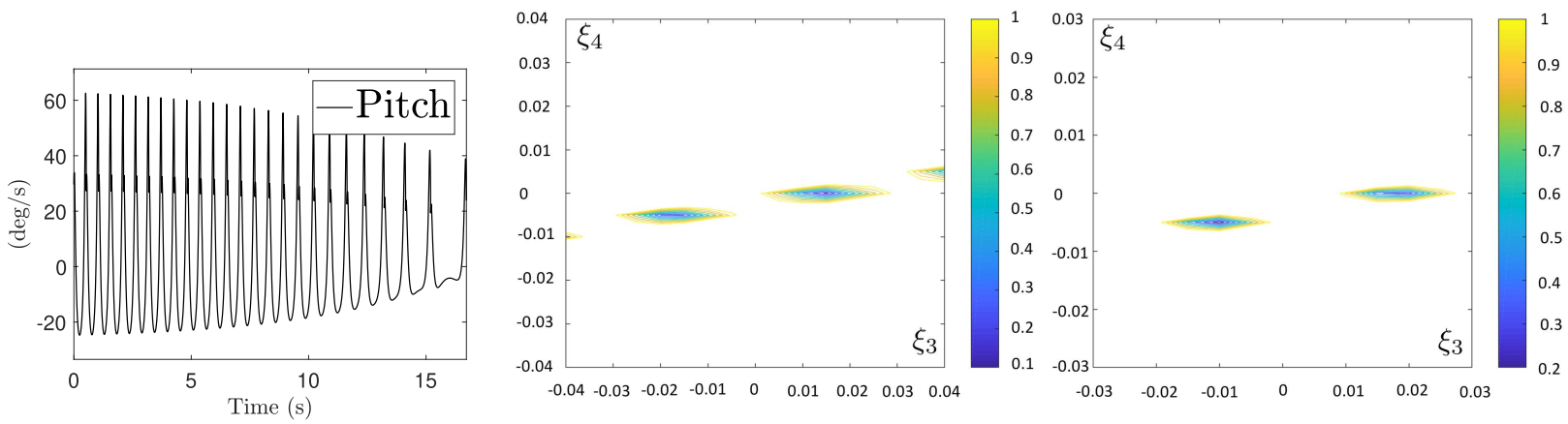

Fig. 3. Plot of the pitch angular velocity versus time during 25 consecutive steps associated with the holonomic output function (left). Here, the initial condition is taken off of the orbit $\mathcal{O}$ and the state trajectory diverges from the orbit. Contour plots of $\max \left(\left|\operatorname{eig}\left(\mathrm{D}_{z} P_{\text {zero }}\left(z^{\star-}\right)\right)\right|\right)$ for the NH-HZD associated with the outputs (7) (middle plot) and (11) (right plot).
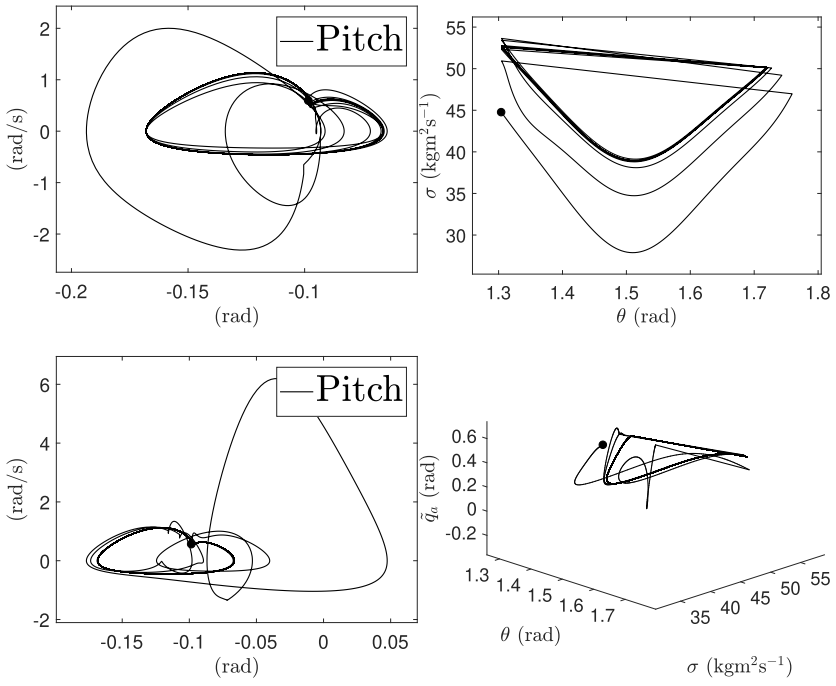

Fig. 4. Phase portraits for the full-order closed-loop system associated with nonholonomic outputs (7) (top) and outputs (11) (bottom) together with the projection of the state solution onto the zero dynamics manifolds $\mathcal{Z}_{\alpha}$ during 100 consecutive steps of planar walking. Convergence to the orbit is clear.

$h_{\sigma}(\sigma)=\xi \sigma \in \mathbb{R}^{4}$, where $\xi:=\operatorname{col}\left(\xi_{1}, \xi_{2}, \xi_{3}, \xi_{4}\right) \in \mathbb{R}^{4}$ denotes the constant parameters to be determined. We will see that the proper selection of the parameter vector $\xi$ is crucial for the stabilization problem. To simplify the analysis, we assume that the first two components of $h_{\sigma}$ that correspond to the stance knee and hip angles are zero, i.e., $\xi_{1}=\xi_{2}=0$. The last two components of $h_{\sigma}$ are then used to control the motion of the swing knee and hip angles. We start with the NH-HZD associated with the relative degree two outputs in (7) and apply Theorem 1 to compute the $1 \times 1$ Jacobian matrix of the restricted Poincaré map. Fig. 3 depicts contour plots of $\left|\mathrm{D}_{z} P_{\text {zero }}\left(z^{\star-}, 0\right)\right|$ to determine the range of stabilizing parameters $\left(\xi_{3}, \xi_{4}\right)$. The white areas in the figure represent the portions of the $\left(\xi_{3}, \xi_{4}\right)$-plane for which the gait is unstable, i.e., the spectral radius of the Jacobian linearization of the Poincaré map is more than 1 . We then study the $2 \times 2$ Jacobian matrix of the restricted Poincaré map for the NH-HZD associated with the relative degree one and two outputs in (11) with the weighting factor $\mu=1$. Fig. 3 also represents the contour plots of the spectral radius of the Jacobian matrix, i.e., $\max \left(\left|\operatorname{eig}\left(\mathrm{D}_{z} P_{\text {zero }}\left(z^{\star-}, 0\right)\right)\right|\right)$ versus $\left(\xi_{3}, \xi_{4}\right)$. For $\mu=0$ that reduces $y_{d 1}$ to the velocity-modulating output, we could not find any stabilizing parameters to stabilize the above-mentioned gait. Hence, we assume that $\mu \neq 0$. From the contour plots of Fig. 3, we choose $\left(\xi_{3}, \xi_{4}\right)=(0.02,0)$ for which the periodic orbit is exponentially stable for the corresponding NH-HZD. Fig. 4 depicts the phase portraits for the full-order closed-loop systems associated with zeroing (7) and (11) together with the projection of the state solution onto the zero dynamics manifolds during 100 consecutive steps of walking. The convergence to the periodic orbit $\mathcal{O}$ is clear. The animation of these simulations can be found in [51].

\section{CONCLUSION}

This brief addressed HZD associated with relative degree one and two nonholonomic outputs for exponential stabilization of given periodic orbits for planar bipedal locomotion. Nonholonomic outputs are constructed such that the associated zero dynamics manifolds contain the orbit while being invariant under both the continuous- and discrete-time dynamics. This brief presented reduced-order models for relative degree one and two NH-HZD. In addition, a computationally attractive framework, based on restricted Poincaré maps, was developed to synthesize NH-HZD controllers that exponentially stabilize underactuated gaits. A comprehensive study of H-HZD and NH-HZD was presented. It was shown that NH-HZD can stabilize a broader range of periodic gaits that are not stabilizable through the application of traditional H-HZD. To illustrate the power of the analytical foundation, this brief numerically verified the NH-HZD framework to stabilize underactuated gaits of a planar bipedal robot. For future research, we will investigate the extension and scalability of the current framework for 3-D bipedal locomotion with high degrees of freedom and underactuation. We will also use this framework to design and experimentally implement stabilizing controllers for underactuated bipedal robots.

\section{ACKNOWLEDGMENT}

The content is solely the responsibility of the authors and does not necessarily represent the official views of NSF.

\section{REFERENCES}

[1] W. Haddad, V. Chellaboina, and S. Nersesov, Impulsive and Hybrid Dynamical Systems: Stability, Dissipativity and Control. Princeton, NJ, USA: Princeton Univ. Press, Jul. 2006.

[2] R. Goebel, R. Sanfelice, and A. Teel, Hybrid Dynamical Systems: Modeling, Stability, and Robustness. Princeton, NJ, USA: Princeton Univ. Press, Mar. 2012.

[3] J. W. Grizzle, G. Abba, and F. Plestan, "Asymptotically stable walking for biped robots: Analysis via systems with impulse effects," IEEE Trans. Autom. Control, vol. 46, no. 1, pp. 51-64, Jan. 2001. 
[4] E. Westervelt, J. Grizzle, C. Chevallereau, J. Choi, and B. Morris, Feedback Control of Dynamic Bipedal Robot Locomotion. Milton Park, Didcot, U.K.: Taylor Francis, 2007.

[5] C. Chevallereau, J. W. Grizzle, and C. L. Shih, "Asymptotically stable walking of a five-link underactuated 3-D bipedal robot," IEEE Trans. Robot., vol. 25, no. 1, pp. 37-50, Feb. 2009.

[6] A. D. Ames, K. Galloway, K. Sreenath, and J. W. Grizzle, "Rapidly exponentially stabilizing control Lyapunov functions and hybrid zero dynamics," IEEE Trans. Autom. Control, vol. 59, no. 4, pp. 876-891, Apr. 2014

[7] M. W. Spong and F. Bullo, "Controlled symmetries and passive walking," IEEE Trans. Autom. Control, vol. 50, no. 7, pp. 1025-1031, Jul. 2005.

[8] M. W. Spong, J. K. Holm, and D. Lee, "Passivity-based control of bipedal locomotion," IEEE Robot. Autom. Mag., vol. 14, no. 2, pp. 30-40, Jun. 2007.

[9] I. R. Manchester, U. Mettin, F. Iida, and R. Tedrake, "Stable dynamic walking over uneven terrain," Int. J. Robot. Res., vol. 30, no. 3, pp. 265-279, Mar. 2011.

[10] H. Dai and R. Tedrake, " $\mathrm{L}_{2}$-gain optimization for robust bipedal walking on unknown terrain," in Proc. IEEE Int. Conf. Robot. Autom., May 2013, pp. 3116-3123.

[11] G. Song and M. Zefran, "Underactuated dynamic three-dimensional bipedal walking," in Proc. IEEE Int. Conf. Robot. Automat., (ICRA), May 2006, pp. 854-859.

[12] R. D. Gregg and L. Righetti, "Controlled reduction with unactuated cyclic variables: Application to 3D bipedal walking with passive yaw rotation," IEEE Trans. Autom. Control, vol. 58, no. 10, pp. 2679-2685, Oct. 2013.

[13] K. Byl and R. Tedrake, "Approximate optimal control of the compass gait on rough terrain," in Proc. IEEE Int. Conf. Robot. Autom., May 2008, pp. 1258-1263.

[14] K. A. Hamed and R. D. Gregg, "Decentralized feedback controllers for robust stabilization of periodic orbits of hybrid systems: Application to bipedal walking," IEEE Trans. Control Syst. Technol., vol. 25, no. 4, pp. 1153-1167, Jul. 2017.

[15] C. Chevallereau et al., "RABBIT: A testbed for advanced control theory," IEEE Control Syst. Mag., vol. 23, no. 5, pp. 57-79, Oct. 2003.

[16] B. Morris and J. Grizzle, "Hybrid invariant manifolds in systems with impulse effects with application to periodic locomotion in bipedal robots," IEEE Trans. Autom. Control, vol. 54, no. 8, pp. 1751-1764, Aug. 2009.

[17] I. Poulakakis and J. W. Grizzle, "The spring loaded inverted pendulum as the hybrid zero dynamics of an asymmetric hopper," IEEE Trans. Autom. Control, vol. 54, no. 8, pp. 1779-1793, Aug. 2009.

[18] K. Sreenath, H.-W. Park, I. Poulakakis, and J. W. Grizzle, "A compliant hybrid zero dynamics controller for stable, efficient and fast bipedal walking on MABEL," Int. J. Robot. Res., vol. 30, no. 9, pp. 1170-1193, Aug. 2011.

[19] S. H. Collins, A. Ruina, R. Tedrake, and M. Wisse, "Efficient bipedal robots based on passive-dynamic walkers," Science, vol. 307, pp. 1082-1085, Feb. 2005.

[20] C. Saglam and K. Byl, "Switching policies for metastable walking," in Proc. IEEE 52nd Annu. Conf. Decis. Control, Dec. 2013, pp. 977-983.

[21] A. M. Johnson, S. A. Burden, and D. E. Koditschek, "A hybrid systems model for simple manipulation and self-manipulation systems," Int. $J$. Robot. Res., vol. 35, no. 11, pp. 1354-1392, 2016.

[22] S. A. Burden, S. S. Sastry, D. E. Koditschek, and S. Revzen, "Eventselected vector field discontinuities yield piecewise-differentiable flows," SIAM J. Appl. Dyn. Syst., vol. 15, no. 2, pp. 1227-1267, 2016.

[23] W. Xi, Y. Yesilevskiy, and C. D. Remy, "Selecting gaits for economical locomotion of legged robots," Int. J. Robot. Res., vol. 35, no. 9, pp. 1140-1154, Aug. 2016.

[24] R. Vasudevan, Hybrid System Identification via Switched System Optimal Control for Bipedal Robotic Walking. Cham, Switzerland: Springer, 2017, pp. 635-650.

[25] H.-W. Park, P. M. Wensing, and S. Kim, "High-speed bounding with the MIT Cheetah 2: Control design and experiments," Int. J. Robot. Res., vol. 36, no. 2, pp. 167-192, 2017.

[26] Y. Hurmuzlu and D. B. Marghitu, "Rigid body collisions of planar kinematic chains with multiple contact points," Int. J. Robot. Res., vol. 13, no. 1, pp. 82-92, Feb. 1994.

[27] A. D. Ames, R. D. Gregg, E. D. Wendel, and S. Sastry, "On the geometric reduction of controlled three-dimensional bipedal robotic walkers," in Lagrangian Hamiltonian Methods for Nonlinear Control. Berlin, Germany: Springer, 2007, pp. 183-196.
[28] R. D. Gregg and M. W. Spong, "Reduction-based control of threedimensional bipedal walking robots," Int. J. Robot. Res., vol. 29, no. 6 , pp. 680-702, May 2010.

[29] A. S. Shiriaev, L. B. Freidovich, and S. V. Gusev, "Transverse linearization for controlled mechanical systems with several passive degrees of freedom," IEEE Trans. Autom. Control, vol. 55, no. 4, pp. 893-906, Apr. 2010

[30] E. R. Westervelt, J. W. Grizzle, and D. E. Koditschek, "Hybrid zero dynamics of planar biped walkers," IEEE Trans. Autom. Control, vol. 48, no. 1 , pp. 42-56, Jan. 2003.

[31] M. Maggiore and L. Consolini, "Virtual holonomic constraints for euler-Lagrange systems," IEEE Trans. Autom. Control, vol. 58, no. 4, pp. 1001-1008, Apr. 2013.

[32] A. Shiriaev, J. W. Perram, and C. Canudas-de-Wit, "Constructive tool for orbital stabilization of underactuated nonlinear systems: Virtual constraints approach," IEEE Trans. Autom. Control, vol. 50, no. 8, pp. 1164-1176, Aug. 2005

[33] A. Mohammadi, M. Maggiore, and L. Consolini, "Dynamic virtua holonomic constraints for stabilization of closed orbits in underactuated mechanical systems," Automatica, vol. 94, pp. 112-124, Aug. 2018.

[34] A. E. Martin, D. C. Post, and J. P. Schmiedeler, "The effects of foot geometric properties on the gait of planar bipeds walking under HZD-based control," Int. J. Robot. Res., vol. 33, no. 12, pp. 1530-1543, 2014.

[35] J. Lack, M. Powell, and A. D. Ames, "Planar multi-contact bipedal walking using hybrid zero dynamics," in Proc. IEEE Int. Conf. Robot. Autom. (ICRA), May 2014, pp. 2582-2588.

[36] A. Hereid, C. M. Hubicki, E. A. Cousineau, and A. D. Ames, "Dynamic humanoid locomotion: A scalable formulation for HZD gait optimization," IEEE Trans. Robot., vol. 34, no. 2, pp. 370-387, Apr. 2018.

[37] A. Ramezani, J. W. Hurst, K. A. Hamed, and J. W. Grizzle, "Performance analysis and feedback control of ATRIAS, a three-dimensional bipedal robot," J. Dyn. Syst., Meas., Control, vol. 136, no. 2, Dec. 2013, Art. no. 021012

[38] C. O. Saglam and K. Byl, "Meshing hybrid zero dynamics for rough terrain walking," in Proc. IEEE Int. Conf. Robot. Autom. (ICRA), May 2015, pp. 5718-5725.

[39] H. Zhao, A. Hereid, W.-L. Ma, and A. D. Ames, "Multi-contact bipedal robotic locomotion," Robotica, vol. 35, no. 5, pp. 1072-1106, May 2017.

[40] R. D. Gregg and J. W. Sensinger, "Towards biomimetic virtual constraint control of a powered prosthetic leg," IEEE Trans. Control Syst. Technol., vol. 22, no. 1, pp. 246-254, Jan. 2014

[41] H. Zhao, J. Horn, J. Reher, V. Paredes, and A. D. Ames, "Multicontact locomotion on transfemoral prostheses via hybrid system models and optimization-based control," IEEE Trans. Autom. Sci. Eng., vol. 13, no. 2, pp. 502-513, Apr. 2016.

[42] A. Agrawal et al., "First steps towards translating HZD control of bipedal robots to decentralized control of exoskeletons," IEEE Access, vol. 5, pp. 9919-9934, 2017.

[43] Q. Cao and I. Poulakakis, "Quadrupedal running with a flexible torso: Control and speed transitions with sums-of-squares verification," Artif. Life Robot., vol. 21, no. 4, pp. 384-392, Dec. 2016.

[44] K. A. Hamed and J. W. Grizzle, "Reduced-order framework for exponential stabilization of periodic orbits on parameterized hybrid zero dynamics manifolds: Application to bipedal locomotion," Nonlinear Anal., Hybrid Syst., vol. 25, pp. 227-245, Aug. 2017.

[45] B. Griffin and J. Grizzle, "Nonholonomic virtual constraints and gait optimization for robust walking control," Int. J. Robot. Res., vol. 36, no. 8, pp. 895-922, Jul. 2017.

[46] A. D. Ames, "First steps toward automatically generating bipedal robotic walking from human data," in Robot Motion Control 2011, K. Kozłowski, Ed. London, U.K.: Springer, 2012, pp. 89-116.

[47] A. D. Ames, "Human-inspired control of bipedal walking robots," IEEE Trans. Autom. Control, vol. 59, no. 5, pp. 1115-1130, May 2014.

[48] J. C. Horn, A. Mohammadi, K. A. Hamed, and R. D. Gregg, "Hybrid zero dynamics of bipedal robots under nonholonomic virtual constraints," IEEE Control Syst. Lett., vol. 3, no. 2, pp. 386-391, Apr. 2019.

[49] A. Isidori, Nonlinear Control System, 3rd ed. Cham, Switzerland: Springer, 1995.

[50] P. de Leva, "Adjustments to Zatsiorsky-Seluyanov's segment inertia parameters," J. Biomech., vol. 29, no. 9, pp. 1223-1230, Sep. 1996.

[51] K. A. Hamed and A. D. Ames. (2018). Nonholonomic Hybrid Zero Dynamics for Stabilization of Periodic Orbits. [Online]. Available: https://youtu.be/AHGIX41tnrw 